\title{
Design of Reasoning Agent for Cognitive Radio Networks
}

\author{
Gyan Vardhan Singh \\ Assistant Professor, Amity University (AUUP) \\ Lucknow Campus, India
}

\begin{abstract}
The main task for a cognitive radio system is to adapt to the transmission parameters in a dynamically changing environment. The nature of wireless channels and the number of transmission parameters to be optimized in various layers add more complexity to the adaptation process. In these situations Case Based Reasoning (CBR) agent along with optimization algorithm provides a robust solution. CBR Agent develops cases as the changes occur in the environment and acquires an understanding of the system. As new situations occur, experience from the previously developed cases is taken into consideration and a solution to the system is provided. In real time with training, the Cognitive Engine (CE) learns the variations occurring in the environment and utilizes the previously existing cases to provide new solution. As well as if abruptly some unexpected situation is encountered then the optimization algorithm is initiated and a solution is designed as per the need of the environment and this case is also added to the case list for future reference. Hence variations in the environment are mapped extensively and the optimization process becomes more efficient. In the present work case based reasoning along with particle swarm optimization is implemented.
\end{abstract}

\section{Keywords}

Artificial Intelligence, Case Based Reasoning, Cognitive Radio Networks, Particle swarm Optimization, Reasoning agent.

\section{INTRODUCTION}

Cognitive Radio (CR) is an intelligent radio which observes the environment, learns from various aspects of it, in order to change its existing parameters in accordance with the needs of the environmental predicates and the quality of service required. Various transmission parameters adapt themselves as per the needs of the external environment. To accomplish the task of cognition, the cognitive engine has been modeled by many researchers world over. Different approaches of artificial intelligence are used to design the optimization and modeling tools for cognitive radio. These approaches have been used for the evaluation process, under different link states. The link state can be represented as an objective in the optimization approach, so as to find the reconfiguration parameters used by CR under current link state. Most of the previous work is done using Genetic Algorithm (GA). In papers [4], [2], [5], [6], Bio-Inspired function for optimization and decision making have been proposed. The focus of these works was mainly on the adaptation and optimization of physical layer parameters, such as transmit power and modulation scheme.

There were many drawbacks of Genetic Algorithms like:

1) It takes long time to find optimum parameters that attain convergence.

2) Fitness value is negatively affected with the increase in the number of objectives.
3) Fitness values are affected negatively as the parameters increase.

It has been seen that Particle Swarm Optimization (PSO) has also been used in cognitive radios to reduce the computational complexity and time of GA. In [7] the comparison of GA and PSO is presented and it is proved that PSO is more computationally efficient. Case Based Reasoning (CBR) along with Adaptive Discrete Particle Swarm Optimization (ADPSO) in turn maintains a potential to be much more computationally efficient and robust to contradicting objectives and provides more extensive solutions.

In this paper, we propose a Reasoning Agent for CR where Case Based Reasoning (CBR) with Adaptive Discrete PSO (ADPSO) algorithm work together to adapt parameters from multiple layers of a CR node. The CBR with PSO gives highly efficient solutions and convergence time is also reduced as cases are developed. Also, ACK (acknowledgement) from receiver is used as a parameter in the adaptation process. The physical layer adaptation parameters (transmit power and modulation scheme) for 16 sub channel system with 3 objectives (minimum BER, maximum data rate, and minimum power consumption) have been considered in the present research

The organization of the paper is as follows: Section 2 gives the overview of case based reasoning and decision making in CR and presents a brief description of ADPSO. Section 3 gives the Algorithmic approach of the CBR based reasoning agent Results are presented in section 4 and section 5 we discuss the conclusion and observations.

\section{CASE BASED DECISION MAKING THEORY AND OPTIMIZATION ALGORITHM}

The CE consists of three major processes:

Orientation

Reasoning, learning \& decision-making; and solution mapping and validity check.

The scenario information is translated into a service request vector in the orientation process. The information on the specific radio environment and the service request are included in service request vector. The reasoning, learning and decision-making process takes the service request vector as input and determines a possible solution. If the anticipated performance of this solution does not satisfy a certain criterion, the solution will be further adapted using adaptation mechanisms until the performance criterion is met or an adaptation time limit is reached. The solution mapping and validity check process maps the solution to operational parameters for specific actions and validates it before applying to the radio [1]. CBR is a reasoning process based on experiences (cases), which resembles human reasoning process to some extent.

One of the advantages of CBR, is that it can work with limited knowledge of the system and provide the solution quickly [4]. This makes CBR better suited for real time application where 
complete pictures of the scenario is not always available and decisions have to be taken by perceiving close approximations to the existing cases. The case based reasoning agent designed in this work proposes solutions based on the cases (experiences) in the case database, revises the old solutions for the new situation, evaluates the performance before applying the solution in the real environment, collects and analyze the feedback information, and updates the case database based on the analysis.

\subsection{Definition of Case}

A case is the fundamental element in a CBR system, it represents an experience. All other components in a CBR system take a case either as input or as output. A case is defined in [4] as follows.

"A case is a contextualized piece of knowledge representing an experience that teaches a lesson fundamental to achieving the goals of the reasoner".

\subsection{Components of CBR}

Definition of Case-based reasoning refers to the reasoning process based on previous recorded experiences (cases). A casebased reasoner is an entity that performs case based reasoning. In general, CBR consists of case representation or case formulation, case selection and retrieval, case evaluation and adaptation, case learning and case database maintenance $[4,2]$.

In case based reasoning the case should be explicit in the following:

- Description of problem: Describes the relevant experience. It specifies the detailed information about the problem including the radio environment and the service request with its QoS (Quality of Service) requirement.

- Solution approach: Explains how the problem was solved in the past. It specifies a possible radio configuration for the problem specified in the problem description.

- Feedback or Outcome or validity of the solution: Records the result of applying the solution. It specifies the feedback from the real environment (e.g., success or failure) after the solution was applied to the customer premise equipment (CPE) [8].

The general structure of the case is given in Fig. 2. The "Problem" specifies the general event (e.g., new customer premise equipment [8] service request, and Primary user (PU) detection, the channel condition (path loss), and the QoS requirement (BER).

The "Solution" includes information on modulation and coding scheme, interleaving, transmission power, and bandwidth use. These are the system parameters the CE can adjust. The "Outcome" includes feedback information (achieved utility).

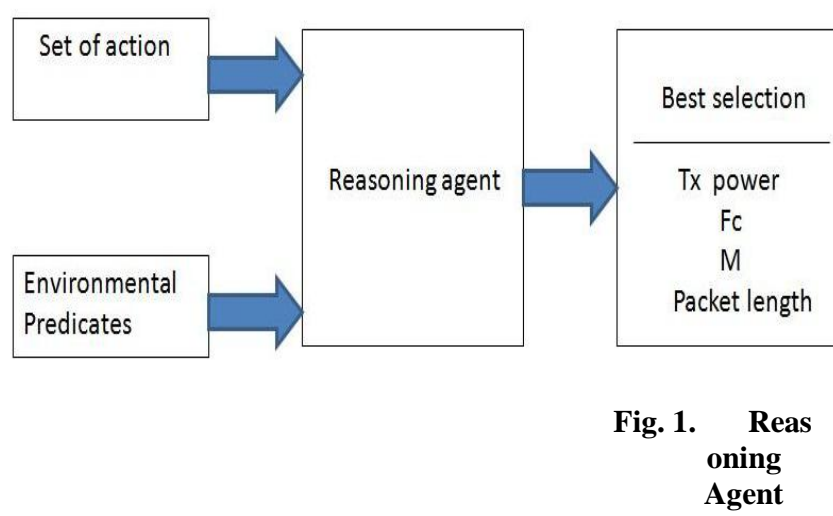

\subsection{Case Selection and Retrieval}

The case selection and retrieval in CBR searches the case database for cases that satisfy the request to a certain extent. Those cases that comply with the policy vector will be collected for further processing. We call them valid cases. The retrieval criterion is the most important aspect in effective case retrieval [2]. For example, shorter Euclidean distance between two situations does not always guarantee a closer match of the two situations. This is why the policy guided retrieval is applied in the Case Based Reasoning - Cognitive Engine (CBR-CE). The case selection and retrieval module returns the best matched case or cases among the valid cases according to a selection criterion. One or multiple valid cases with highest utility are returned. The choice of the selection criterion is an open question in many CBR applications. The utility metric we use is a choice with acceptable performance. Other metrics in utility functions such as those discussed in [3] can be adopted easily in this process of designing the CBR. For example, it is easy to implement case selection functionality with a metric on transmission power, for example selecting a case with the minimum transmission power.

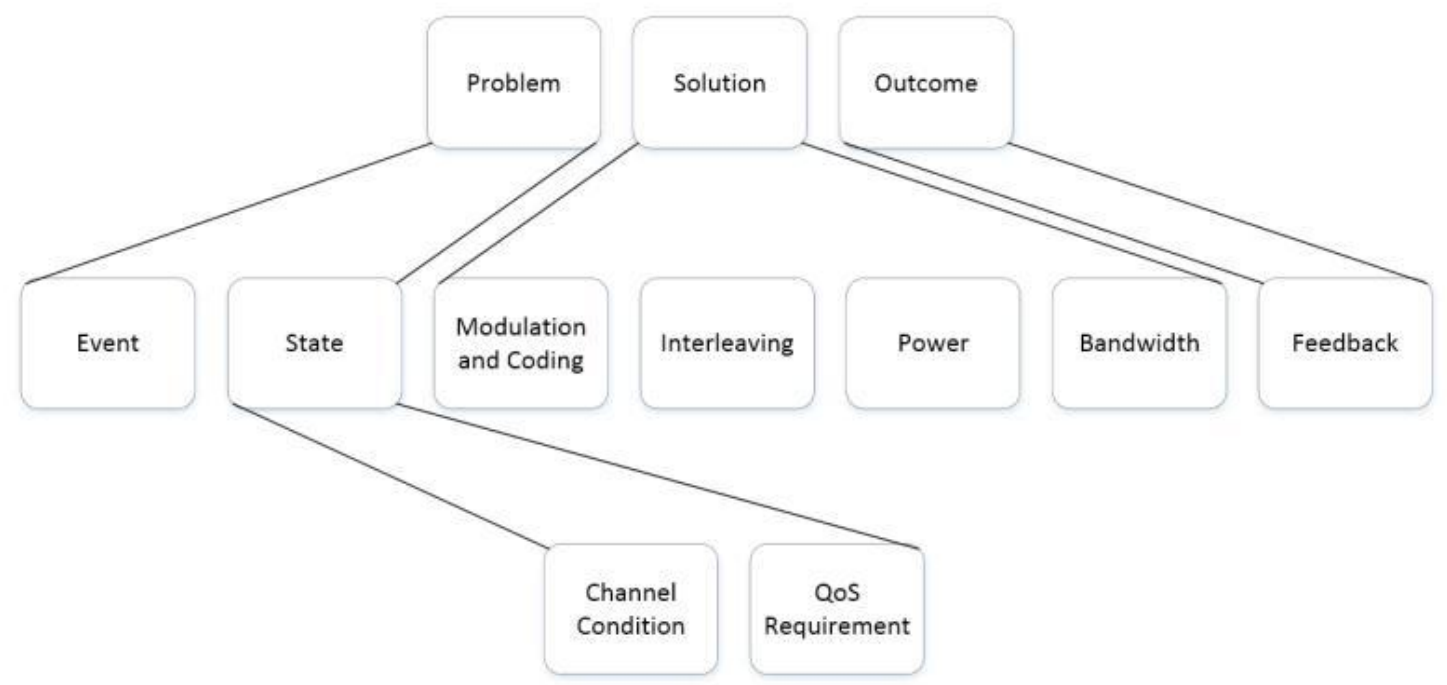

Fig 2. Case Structure [1] 


\subsection{Case Evaluation and Adaptation}

The case evaluation module evaluates the performance of the retrieved case from the case selection and retrieval module either by using a performance model or by applying the solution and observing the outcome. The retrieved case is expected to be applicable to the new problem. However, the applicability or the performance is not guaranteed. The retrieved case is recorded as a solution to a previous problem and retrieved as a possible solution to the new problem due to the similarity between the old problem and the new one. If the performance of the retrieved case is not satisfactory, the case is modified by the case adaptation module. Note that the case evaluation and case adaptation can be recursive in order to obtain an appropriate solution. It increases the processing time. Therefore, it is employed only if it is needed.

\subsection{Case Learning and Case Library}

A case library is an ensemble of similar cases. Several case libraries can be organized in a hierarchical structure into a case database for efficient retrieval. Experiences are remembered by the CBR system as cases in the case database. The CBR gains additional information, or learns, by solving new problems or receiving feedback. As the experience increases, more cases are accumulated in the case database. New experience may also be incorporated in the case database by updating the existing cases. In this way, the size of the case database will not increase linearly with the number of new problems and may finally converge. Although a larger case database does not always guarantee better performance for a specific problem, a CBR system can generally return a better solution with a larger case database than with a smaller one. This performance improvement reflects the learning capability of a CBR system. The case database maintenance module is critical to the learning process and reasoning process of the CBR-CE. When a solution to a new problem is formulated, this solution needs to be "remembered" by the CBR for future use. The outcome of applying this solution to the new problem should be "remembered", too. As more and more cases are recorded in the case database, the CBR gets more and more efficient in the sense that it can utilize cases in the case database to the new problem instead of finding a solution from scratch using computationally intensive optimization. Another important consideration in case database maintenance is the balance between the number of cases in the case database and the amount of time used in case retrieval. A larger case database covers a larger problem space. However, this usually also means a longer case retrieval time and larger memory space. Although memory size is becoming less of a concern in modern system design due to advancements in memory size, processing time is always a big concern in many wireless applications. Therefore, an appropriate case granularity needs to be defined and redundant cases removed from the case database to improve execution time [1].

\subsection{Optimization Algorithm: Particle Swarm Optimization (PSO)}

In PSO, a set of random solutions (initial swarm) is generated. This swarm is distributed in the design space towards the optimal solution over a number of iterations (moves) based on large amount of information about the design space that is assimilated and shared by all members of the swarm. Adaptation and optimization in $\mathrm{CR}$ is the process of determining the best transmission parameters that achieve QoS requirements, under specific constraints. The Cognitive Engine (CE) uses the optimization algorithm to find this optimal set of transmission parameters, and to achieve the best value of the desired objective $(O b j)$. The best objective value corresponds to the best fitness $(f)$ value. The optimization algorithm has the following equation:

$$
f_{\max }=\operatorname{Obj}(\vec{a})
$$

Where $\vec{a}=\left[a_{1}, a_{2}, \ldots, a_{n}\right]$ denote the set of adapted parameters to achieve desired objective (transmit power, modulation scheme, carrier frequency, packet length, etc). Many objectives represent the link states such as minimum bit error rate, maximum data rate, maximum throughput, minimum power consumption and maximum spectral efficiency. The data rate and bit error rate are very important to ensure required QoS and power consumption is very important objective in green cognitive radio application.

The main aims of the optimization and adaptation algorithm are:

- Reducing the time taken to converge while finding the optimal set of parameters for CR in multi-carrier system with multiple-objectives in a time varying channel.

- Overcoming local optimum problem in DPSO and GA. To achieve this objective, ADPSO converts each parameter in the particle; to binary format with a specified number of bits to represent the whole search space of each parameter (setting the correct number of bits is an important task, because the search space depends on how we choose to represent the parameters in binary format).

In our case based approach for designing a reasoning agent we use ADPSO (Adaptive Discrete Particle Swarm Optimization) [9]. The main aim of the work is to use case base theory to approximate the environmental variation completely and use the existing cases in making decision for new environmental changes, whereas the optimization algorithm runs for some instances but after sufficient training, enough experience is gained regarding the behavior of the environment. In our approach we try to minimize the initiation of adaptation algorithm for every instance of environment change, rather after some training the case based algorithm gains enough experience in terms of various cases to model the new demands of the environment and QoS.

\section{ALGORITHMIC APPROACH OF THE CBR BASED REASONING AGENT}

In a case based system, solutions to varying conditons of environment, QoS or user requirements are saved as cases. These cases are utilized to approximate a solution when a similar case occurs. In this paper we first define three different scenarios:

- First Scenario is the case where the QoS requirement of the communication is given importance and accordingly the Optimization algorithm adapts itself to provide cases which suffice the requirement.

- The second scenario takes care of the fact when low power transmission is required.

- Third scenario incorporates the conditions where high bit rate transmission is required and forces the optimization process to deliver according to the high bit rate constraints. 


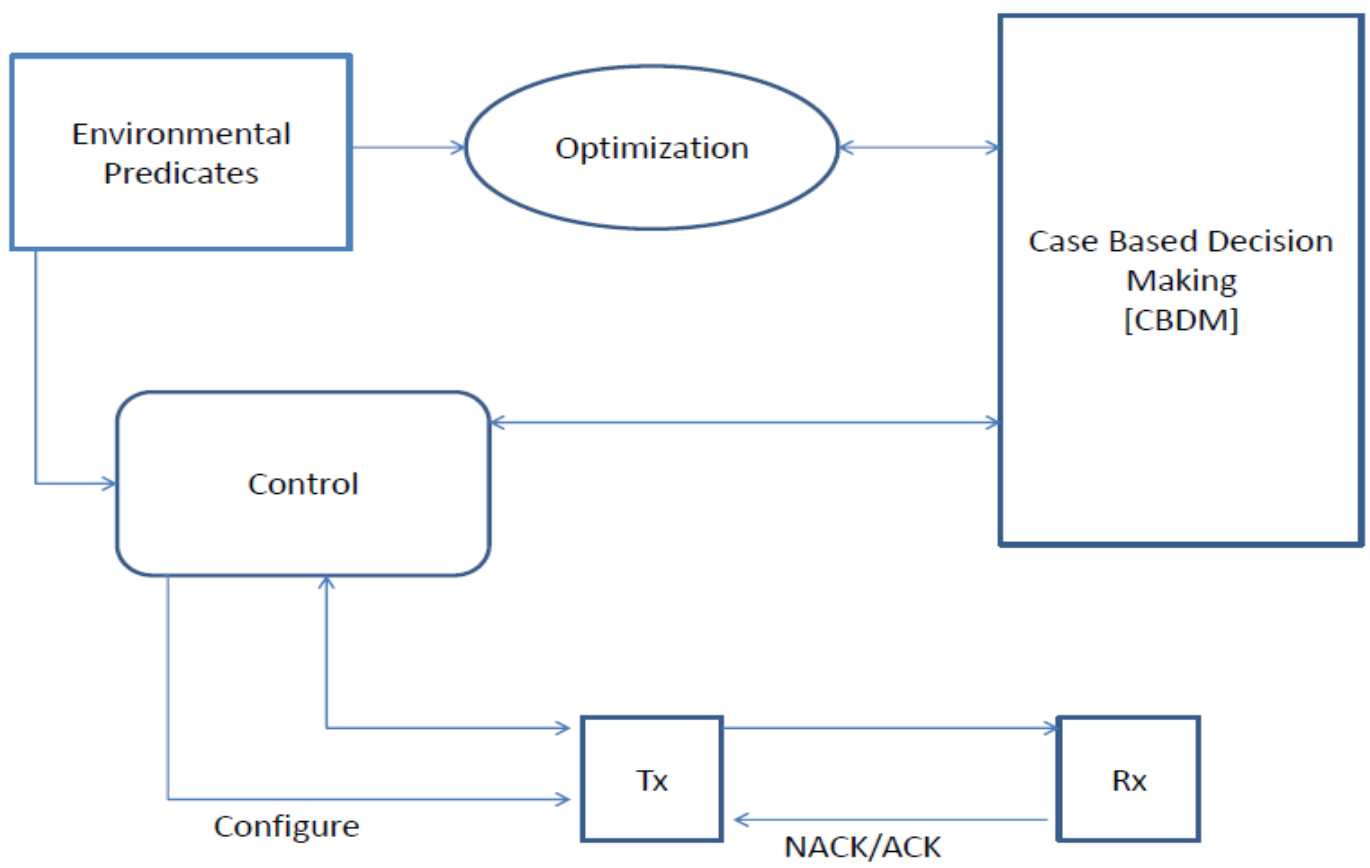

Fig. 3: Case Based Decision Making with Adaptive Discrete Particle swarm Optimization

In rule based systems based on case based decision making the optimization algorithm works in the background and the case based system handles most of the load. This approach decreases the convergence time required to reach a solution and results in an better optimized performance. In the present work $16 \mathrm{sub}$ channel system is considered and three scenarios have been defined as explained earlier. Each case stored in the case base is constituted of complete description of the environmental parameters, fitness value, feedback or validity of the solution, solution in terms of modulation, packet length, coding, transmitt power etc. As solutions are provided on multiple objective adaptation hence we can add more explicit parameter details which make the approximation more accurate.

In the case based approach, initially as the external parameters change adaptation algorithm provides solutions accordingly. These solutions are validated and if the performance creteria as per demands of scenario is met then an acknowledgement (ACK) is sent. The solution along with feedback and objectives is stored in the case base as a case, likewise if an negative acknowledgement (NACK) is received then that case is also stored and adaptation mechanisms performs further optimization till an optimized solution is achieved which meets the performance criteria or an adaptation time limit is reached. This is the infancy stage of the case based system and after some training it gets experienced with cases and understands the environment. Further when CR senses environmental change, the CBR approximates a solution on the basis of previously existing cases and sends it.

An ACK or NACK is generated accorded as per the satisfaction of the requirement. If the feedback is NACK then the CR learns that the solution approximated by the CBR is not optimized and it calculates the optimized solution through ADPSO [9].

In real time with little training the CBR-CE learns about the environment and the requirements from the past experiences and aproximation grows better and the optimization process is greatly improved with very low convergence time.

\section{RESULTS}

The approach proposed in this paper is considered for three different scenario, refer Table 1. For each scenario there is a specific weight to each objective function, different scenarios have specific performance objective, with specific QoS requirements and accordinly the weights are assigned. The total fitness function is determined by:

$$
f_{\text {total }}=w_{1} f_{\text {min power }}+w_{2} f_{\max R_{b}}+w_{3} f_{\min B E R}
$$

The simulation results for CBR reasoning agent in CR under the scenario (Low Power) is shown in Fig. 4a \& Fig. 4b. This is the trial run for Low power scenario and for a case base of 20 cases; the approximation of case based reasoning is evaluated. After every trail run the cases were saved in the rule base and that rule base served as the basis for the next run. One more approach which is followed is that some generic case base is created with respect to general environmental changes obtained from domain specific knowledge. Hence before the trials are initiated a pre existing rule base of 20 cases is already existing and during the run the rule base updated itself with scenario based cases. Also as the trials are increased we notice that the ACK goes for a linear rise and NACK lowers down, this was a small trials. Combined with PSO the probability of getting a NACK would be very low. One more thing which is worth noticing is as the training goes the convergence time of the system decreases sharply.

Table 1: Scenario

\begin{tabular}{|c|c|c|c|}
\hline Scenario & $\begin{array}{c}\text { High Bit } \\
\text { Rate }\end{array}$ & Low BER & Low Power \\
\hline Weight $\left(w_{1}\right)$ & 0.05 & 0.05 & 0.8 \\
\hline Weight $\left(w_{2}\right)$ & 0.15 & 0.8 & 0.15 \\
\hline Weight $\left(w_{2}\right)$ & 0.8 & 0.15 & 0.05 \\
\hline
\end{tabular}




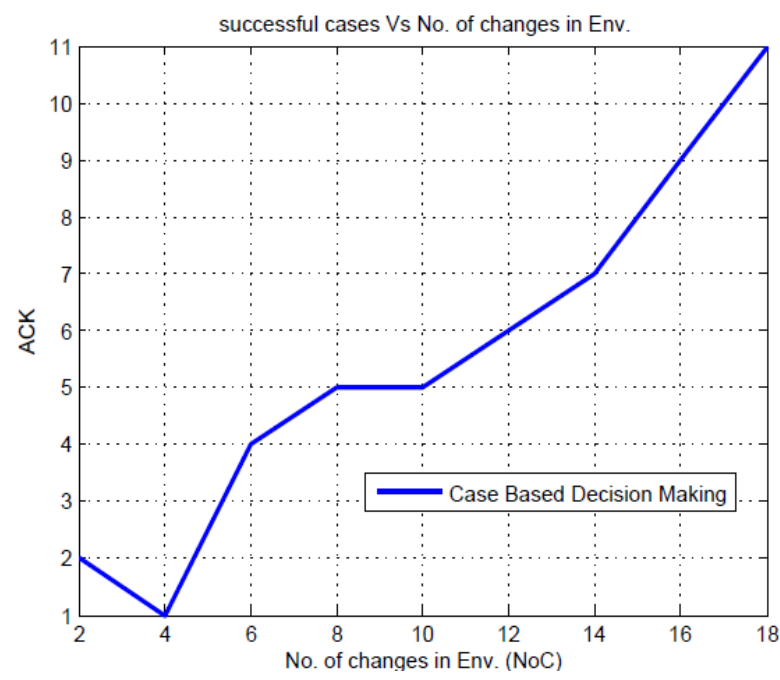

Fig. 4a: Successful cases

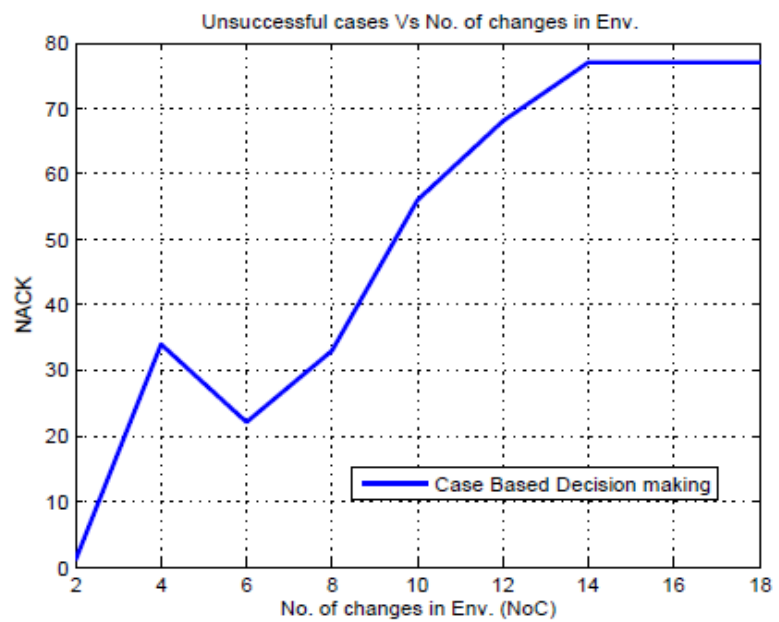

Fig. 4b: Unsuccessful cases

\section{CONCLUSION AND OBSERVATION}

In real time application quick convergence time as well as optimized solution is very crucial in order to maintain the link between the two nodes and the QoS. The Reasoning agent based on case based reasoning works in two ways, firstly it watches the environment and its behavior and helps in optimization process to adapt more closely to the environment in order to fulfill the performance criteria and secondly as explicit training is attained the case based system becomes experienced enough with the help of previous cases to approximate closely to an optimal solution without initiating the extensive procedures to calculate the solution.

\section{ACKNOWLEDGEMENT}

This work corresponds to my research at TU Ilmenau. My thanks to Integrated Communication Systems Group (ICS) of Technische Universität Ilmenau, Germany for giving me the opportunity to work on this project. And also thanks to University computer center for extending their cluster computing facilities.

\section{REFERENCES}

[1] A.He, J.Gaeddert, K. K. Bae, J. H. Reed, and C. H. Park, "Development of a Case-Based Reasoning Cognitive Engine for IEEE 802.22 WRAN Applications", IEEE Transactions, 2007.

[2] S. Shiu and S. K. Pal, Foundations of Soft Case-Based Reasoning, Wiley Series on Intelligent Systems.WileyInterscience, Hoboken, NJ.

[3] T.R. Newman, A. M. Wyglinski, A. Agah, J. B. Evans, and G. Minden, "Cognitive engine implementation for wireless multicarrier transceiver", Wireless Communication and Mobile Computing, 7(9):1129-1142, 2007.

[4] J. L. Kolodner and D. Leake, A tutorial introduction to case-based reasoning, Case-Based Reasoning: Experiences, Lessons and Future Directions, chapter 2, pages 31-65.MIT Press, Cambridge, MA, 1996.

[5] J. H. Reed et al. Development of a cognitive engine and analysis of WRAN cognitive radio algorithms phase II Report submitted to ETRI, MPRG, Virginia Tech, December 2006.

[6] B. Le, T.W. Rondeau, and C.W. Bostian, "Cognitive radio realities", Wireless Communication and Mobile Computing, 7(9):1037-1048, 2007.

[7] T. W. Rondeau, B. Le, C. J. Rieser, and C. W.Bostian, "Cognitive radios with genetic algorithms: Intelligent control of software defined radios," in SDR Forum Technical Conference, pages C-3-C-8, Phoenix, AZ, 2004

[8] An He et. al. "Survey Of Artificial Intelligence For Cognitive Radios", IEEE Transactions On Vehicular Technology, Vol. 59, No. 4, May 2010.

[9] Mahdi, A.H.; Mohanan, J.; Kalil, M.A.; Mitschele-Thiel, A., "Adaptive Discrete Particle Swarm Optimization for Cognitive Radios," in Communications (ICC), 2012 IEEE International Conference on, vol., no., pp.6550-6554, 1015 June 2012. 